\title{
Tratamiento endovascular de la enfermedad obstructiva aortoilíaca
}

\author{
Luis C. Hernández-Moreno ${ }^{1,2}$, Angela G. Poblete-Saavedra, ${ }^{1,2}$, \\ Alejandro J. Kotlik-Aguilera ${ }^{1,2}$ y Juan R. Bombin-Franco ${ }^{1,2}$
}

\section{Endovascular management of aortoiliac occlusive disease}

Introduction: Percutaneous transluminal angioplasty (PTA) has become an accepted technique in the treatment of aortoiliac occlusive disease, with success rates of $90-92 \%$, and primary patency of 55 $72 \%$ at 5 years. Aim: To evaluate the results of endovascular treatment (PTA or PTA with stents) of the aortoiliac sector. Material and Method: Descriptive, retrospective study (single-center case series) of patients, consecutively subjected to endovascular treatment (PTA or PTA with stents) of aortoiliac occlusive disease, during a period of 7 years (2002 - 2019), at the Dr. Eduardo Pereira Hospital in Valparaíso, Chile. Results: 103 procedures were performed in 94 patients, male: $63.83 \%$, female: $36.17 \%$, mean age: 67.4 years (range 47-96), distribution of the lesions according to the TASC II classification: A (46.24\%), B (39.78\%), C (8.60\%), D (5.38\%), remodeling the aortic bifaction (kissing stent) in $6.80 \%$, hybrid procedures (12.62\%), average follow-up (47.13 months), clinical success $(90.29 \%)$, technical success $(94.17 \%)$, primary patency, assisted primary and secondary at 5 years of $68.09 \%, 75,53 \%$ and $81.91 \%$ respectively, 5-year limb salvage rate of $84.04 \%$, mortality $<30$ days of $1.94 \%$, 5-year survival of $90.42 \%$. Discussion: Endovascular techniques in the aortoiliac sector are reliable, their technical results and patency are influenced by the clinical stage of the patient and the severity of the lesions treated. Conclusion: In appropriately selected patients, endovascular treatment of the aortoiliac sector, presents excellent results, allowing an increase in the indication for treatment in patients considered to be at high risk.

Keywords: iliac angioplasty; iliac stenting; iliac artery occlusion; aortoiliac occlusive disease.

\section{Resumen}

Introducción: La angioplastia transluminal percutánea (ATP), se ha convertido en una técnica aceptada, en el tratamiento de la enfermedad obstrutiva aortoilíaca, con tasas de éxito del 90-92\% y permeabilidad primaria del 55-72\% a 5 años. Objetivo: Evaluar los resultados del tratamiento endovascular del sector aortoilíaco. Material y Método: Estudio descriptivo, retrospectivo (revisión de serie de casos unicéntrica), de pacientes, sometidos consecutivamente al tratamiento endovascular (ATP simple y ATP con stent) de la patología obstructiva del sector aortoilíaco, durante un período de 7 años (2002-2019), en el Hospital Dr. Eduardo Pereira de Valparaíso, Chile. Resultados: Se realizaron 103 procedimientos en 94 pacientes, sexo masculino: 63,83\%, femenino: 36,17\%, edad promedio: 67,4 años (rango 47-96), distribución de las lesiones según la clasificación TASC II: A $(46,24 \%)$, B $(39,78 \%)$, C (8,60\%), D $(5,38 \%)$, remodelando la biburfaccción aórtica (kissing stent) en un 6,80\%, procedimientos híbridos (12,62\%), seguimiento promedio (47,13 meses), éxito clínico (90,29\%), exito técnico (94,17\%), permeabilidad primaria, primaria asistida y secundaria a 5 años del 68,09\%, 75,53\% y $81,91 \%$ respectivamente, tasa de salvación de la extremidad a 5 años del $84,04 \%$, mortalidad $<30$ días del 1,94\%, supervivencia a 5 años del 90,42\%. Discusión: Las técnicas endovasculares del sector aortoilíaco son fiables, sus resultados ténicos y permeabilidad, están influenciados por el estadio clínico del paciente y severidad de las lesiones tratadas. Conclusión: En pacientes adecuadamente seleccionados, el tratamiento endovascular del sector aortoilíaco presenta excelentes resultados, permitiendo aumentar la indicación de tratamiento en pacientes considerados de alto riesgo.

Palabras clave: angioplastía ilíaca; stent ilíaco; oclusión ilíaca; aterosclerosis aortoilíaca.
'Servicio de Cirugía Hospital Dr. Eduardo Pereira Ramírez. Departamento de Cirugía. Universidad de Valparaíso. Valparaíso, Chile.

Recibido 2020-08-24 y aceptado 2020-10-13

Correspondencia a: Dr. Luis C. Hernandez-Moreno luiscesarh@gmail.com 


\section{Introducción}

Aproximadamente el 3 a $20 \%$ de la población mundial cercana a los 70 años, presenta enfermedad arterial obstructiva de extremidades inferiores ${ }^{1,2}$, donde el sector aortoilíaco representa el 35\% de estos casos, y el segundo afectado en frecuencia después del femoropoplíteo ${ }^{2,3}$.

El consenso TASC II ${ }^{4}$, y las Guías AHA/ACC 5 para el manejo de la enfermedad arterial periférica, proponen que las lesiones aortoilíacas tipo A y B se benefician del tratamiento endovascular, mientras las lesiones más extensas (C y D), se benefician del tratamiento quirúrgico convencional.

Sin embargo, el perfecionamiento progresivo de los dispositivos endovasculares (menor perfil, mayor flexibilidad y resistencia), asociado al mejor conocimiento de su comportamiento a largo plazo, y desarrollo de técnicas híbridas (procedimiento endovascular asociado a cirugía convencional), permiten el progresivo aumento del tratamiento endovascular de lesiones tipo $\mathrm{C}$ y excepcionalmente $\mathrm{D}$, con resultados aceptables ${ }^{6-9}$.

El tratamiento híbrido, esta indicado en el grupo de pacientes con afectación multifocal y elevada morbilidad $^{10}$, permitiendo disminuir riesgos y costos asociados $^{11}$, con tasas de éxito técnico superiores al $90 \%$, y salvamento de la extremidad del $95 \%$ a 2 años $^{10,12}$.

La eficacia del tratamiento de las lesiones ilíacas con la angioplastía transluminal percutánea (ATP), está ampliamente aceptado ${ }^{13}$. La experiencia global evidencia un éxito técnico del $90-92 \%{ }^{14,15}$, permeabilidad primaria del $75-90 \%$ al año, y del $55-72 \%$ a 5 años ${ }^{14-16}$.

El propósito del presente estudio, es evaluar los resultados del tratamiento endovascular (ATP simple o con stent), de las lesiones obstructivas del sector aortoilíaco, en pacientes intervenidos en el Hospital Dr. Eduardo Pereira de Valparaíso, Chile.

\section{Metodología}

Estudio descriptivo, retrospectivo (revisión de serie de casos unicéntrica), de los registros clínicos y base de datos de pacientes, sometidos consecutivamente al tratamiento endovascular de las lesiones obstructivas del sector aortoilíaco, durante un período de 7 años (2002-2019), en el Hospital Dr. Eduardo Pereira de Valparaíso, Chile.

La categorización clínica de los pacientes se realizó utilizando la clasificación de Rutherford modificada, definiendo la anatomía de la lesión mediante la clasificación TASC II (Figura 1). Las indicaciones (criterios de inclusión), y contraindicaciones (criterios de exclusión), para realizar el procedimiento se describen en la Tabla 1.

Las técnicas empleadas fueron: ATP simple y ATP con stent. Los stent utilizados corresponden a las marcas comerciales: Absolute ${ }^{\circledR}$, Absolute Pro $^{\circledR}$ (Abbott Vascular, EE. UU), Epic ${ }^{\mathrm{TM}}$ y Wallstent ${ }^{\mathrm{TM}}$ (Boston Scientific, EE. UU), Everflex ${ }^{\mathrm{TM}}$ (Medtronic, EE .UU) y Astron ${ }^{\circledR}$ (Biotronik, SUI).

El éxito clínico se definió como la mejoría en al menos un grado de la clasificación de Rutherford, y el éxito técnico como la revascularización de la lesión con estenosis residual $<30 \%$, en ausencia de disección que comprometa el flujo, con implantación apropiada en caso de utilizar stent.

La permeabilidad primaria se definió como el tiempo que el segmento reparado, permaneció permeable sin reestenosis $\geq 50 \%$ o necesidad de intervención adicional. La permeabilidad primaria asistida, como el tiempo que el segmento reparado encontrandose permeable, ameritó una intervención adicional para mejorar su permeabilidad, y la permeabilidad secundaria como el tiempo transcurrido desde la primera ATP aortoilíaca, hasta que la reparación experimentó oclusión o fracaso hemodinámico, finalizando el seguimiento al producirse una oclusión no tratable o fallecimiento del paciente.

Las variables principales evaluadas corresponden al éxito clínico y técnico del procedimiento, permeabilidad, salvación de la extremidad y supervivencia. Las variables secundarias estudiadas fueron sexo, edad, estadío clínico, morbilidad, sangrado y estancia hospitalaria.

Nuestra investigación al ser un estudio retrospectivo, unicentrico, se encuetra sujeta a potenciales sesgos de selección, relacionado a la existencia de diferentes tipos de lesiones ilíacas y estadíos clínicos. Otro sesgo importante corresponde al análisis de la permeabilidad global de la ATP aortoilíaca, aplicando dos técnicas de reparación diferentes (ATP simple vs ATP con stent), en una población heterogenea.

Las intervenciones fueron efectuadas en su mayoría con anestesia local o raquídea, y en casos excepcionales anestesia general, utilizando siempre como abordaje la arteria femoral común, administrando intraprocedimiento heparina no fraccionada sistémica, a dosis de $1 \mathrm{mg} / \mathrm{kg}$ de peso corporal.

Las indicaciones para la implantación de un stent fueron: retroceso elástico posterior a la ATP, disección amplia, contornos irregulares sugerentes de vía subintimal, flap intimal, oclusión, estenosis residual 


Lesiones tipo A:
- Estenosis unilateral o bilateral de la AIC
- Estenosis unilateral o bilateral única corta $(<3 \mathrm{~cm})$ de la AlE
Lesiones tipo B:
- Estenosis corta $(<3 \mathrm{~cm})$ de la aorta infrarrenal
- Oclusión unilateral de la AIC
- Estenosis única o múltiples de 3 -10 cm que incluye la AlE sin com-
promiso de la AFC
- Oclusión unilateral de la AIE que no compromete el origen de la All
ni la AFC
Lesiones tipo C:
- Oclusión bilateral de la AIC
- Estenosis bilateral de la AIE de 3-10 cm sin compromiso de la AFC
- Estenosis unilateral de la AIE que compromete la AFC
- Oclusión unilateral de la AIE que compromete el origen de la All y/o
la AFC
- Oclusión severamente calcificada unilateral de la AIE con o sin com-
promiso del origen de la All y/o de la AFC
Lesiones tipo D:
- Oclusión aorto-ilíaca infrarrenal
- Enfermedad difusa de la aorta infrarenal y ambas ilíacas
- Estenosis difusa con compromiso unilateral de la AIC, AIE, AFC
- Oclusión unilateral de la AIC y AlE
- Oclusión bilateral de la AlE
- Estenosis ilíaca en paciente con AAA que requiere tratamiento qui-
rúrgico no endovascular

Figura 1. Clasificación TASC II, para las lesiones del sector aortoilíaco. AAA: aneurisma de aorta abdominal, AIC: arteria ilíaca común, AlE: arteria ilíaca externa, AFC: arteria femoral común
$>30 \%$, reestenosis, estenosis larga, lesiones calcificadas y/o excéntricas, lesiones ulceradas (riesgo de ateroembolismo).

Recibiendo todos los pacientes durante los 6 meses posteriores al procedimiento, doble antiagregación plaquetaria con ácido acetilsalisílico (AAS) y clopidogrel, manteniendo posteriormente AAS a permanencia.

En todos los pacientes se realizó seguimiento clínico ambulatorio y con ultrasonido doppler, a los 1, 6, 12 meses y posteriormente anual, asociando angiotomografia o arteriografía selectiva, en caso de evidenciar complicaciones clínicas o estenosis $\geq$ $50 \%$ en el ultrasonido doppler, con indicación para reintervenir al evidenciar estenosis $\geq 70 \%$ o deterioro significativo del estatus clínico.

El análisis estadístico se realizó aplicando las pruebas de Chi cuadrado, test de Fisher, utilizando t de Student para la comparación de variables, y tablas de supervivencia de Kaplan-Meier para el análisis de la permeabilidad, considerando un valor de $\mathrm{p} \leq 0,05$ como estadísticamente significativo.
Tabla 1. Indicaciones y contraindicaciones para realizar la ATP del sector aortoilíaco

Indicación
- Pacientes estables
- Estadio clínico $\geq 3$ de la clasificación de Rutherford
- Lesiones TASC II A y B
- Pacientes con elevada morbilidad y contraindicación para realizar cirugía
convencional, con lesiones TASC II C y D
Contraindicación
Absoluta
- Estadio clínico 1 y 2 de la clasificación de Rutherford
- Patología que amenaza con infectar la endoprótesis (infección, bacteremia,
sepsis)
- Imposibilidad para realizar seguimiento a mediano y largo plazo
- Sensibilidad o alergia conocida a los materiales de la endoprótesis
Relativa
- Sensibilidad o alergia conocida al medio de contraste
- Enfermedad renal crónica avanzada
- Tortuosidad y ateromatosis severa del sector ilíaco




\section{Resultados}

Se realizaron 103 procedimientos en 94 pacientes, sexo masculino $63,83 \%$, femenino $36,17 \%$, edad promedio 67,4 años (rango 47-96), los factores de riesgo para desarrollar enfermedad vascular (Tabla $2)$, corresponden a hipertensión arterial $(74,47 \%)$, tabaquismo $(69,14 \%)$ y diabetes mellitus $(67,02 \%)$.

La categoría clínica de Rutherford previa al procedimiento (Tabla 2) fue: C3: 48,93\%, C4: $34,04 \%$, C5: 9,57\%, C6: 6,38\%. La distribución de las lesiones según la clasificación TASC II (Tabla 3) fue: A $(46,24 \%), \mathrm{B}(39,78 \%), \mathrm{C}(8,60 \%), \mathrm{D}(5,38 \%)$.

Tabla 2. Características basales de la población estudiada $(n=94)$

\begin{tabular}{|lcc|}
\hline & $\mathbf{n}$ & $\mathbf{\%}$ \\
Genero & & \\
Masculino & 60 & 63,83 \\
Femenino & 34 & 36,17 \\
Comorbilidades asociadas & & \\
Hipertensión arterial & 70 & 74,47 \\
Tabaquismo & 65 & 69,14 \\
Diabetes mellitus & 63 & 67,02 \\
Dislipidemia & 45 & 47,87 \\
Obesidad (IMC > 30) & 21 & 22,34 \\
Cardiopatía coronaria. & 15 & 15,95 \\
Enfermedad pulmonar obstructiva crónica & 14 & 14,89 \\
Enfermedad cerebrovascular & 12 & 12,76 \\
Enfermedad renal avanzada & 8 & 8,51 \\
Presentación clínica (clasificación Rutherford). & & \\
0: Asintomático & 0 & 0 \\
1: Claudicación leve & 0 & 0 \\
2: Claudicación moderada & 0 & 0 \\
3: Claudicación severa & 47 & 48,93 \\
4: Dolor isquémico en reposo & 32 & 34,04 \\
5: Perdida tisular menor & 9 & 9,57 \\
6: Perdida tisular mayor & 6 & 6,38 \\
\hline
\end{tabular}

Tabla 3. Tasa de éxito técnico y permeabilidad primaria global, de la angioplastía del sector aortoilíaco, relacionado a la clasificación TASC II

\begin{tabular}{|cccccc|}
\hline $\begin{array}{c}\text { Clasificación } \\
\text { TASC II }\end{array}$ & n (\%) & Tasa de & \multicolumn{3}{c|}{ Permeabilidad primaria (\%) } \\
\hline A & $43(46,24)$ & $42(97,67)$ & 95,34 & 86,04 & 74,42 \\
B & $38(39,78)$ & $36(94,73)$ & 92,10 & 78,94 & 68,42 \\
\hline C & $8(8,60)$ & $7(87,5)$ & 87,5 & 62,5 & 50 \\
\hline D & $5(5,38)$ & $4(80)$ & 80 & 60 & 40 \\
\hline Total & $94(100)$ & $89(94,68)$ & 92,55 & 79,78 & 68,09 \\
\hline
\end{tabular}

Afectando a la ilíaca común $(32,26 \%)$, iliaca externa (39,78\%), y simultáneamente ambas ilíacas $(27,96 \%)$, donde el $82,98 \%$ corresponde a estenosis, y $17,02 \%$ a lesiones oclusivas. Utilizando siempre como abordaje la arteria femoral común, vía ipsilateral $(86,40 \%)$, contralateral $(13,6 \%)$, realizando la ATP de lesión única en el 76,6\%, dos lesiones en el $17,02 \%$, y tres lesiones en el $6,38 \%$, implantado un promedio de 1,43 stent.

Realizando la remodelación de la bifurcación aórtica (kissing stent) en un 6,80\% (7 casos), y procedimientos híbridos en un $12,62 \%$ (13 casos): bypass femoro femoral cruzado $(46,15 \%)$, bypass fémoro-poplíteo $(30,77 \%)$, endarterectomía femoral común $(15,38 \%)$, bypass femoro tibial $(7,69 \%)$.

En relación a la clasificación TASC II, las lesiones tipo A y B, presentaron la mejor tasa de éxito técnico, $97,67 \%$ y $94,73 \%$ respectivamente. Presentando las lesiones tipo $\mathrm{C}$ y D, las tasas más bajas de éxito técnico, $87,5 \%$ y $80 \%$ respectivamente (Tabla 3).

De igual forma las lesiones tipo A y B, presentaron la mejor permeabilidad primaria a 5 años, $74,42 \%$ y $68,42 \%$ respectivamente. Presentando las lesiones tipo $\mathrm{C}$ y D, la permeabilidad primaria más baja a 5 años, 50\% y 40\% respectivamente (Tabla $3)$.

Obteniendo una tasa de éxito técnico global de la ATP aortoilíaca (Tabla 4) del 94,17\%, con un $2,91 \%$ ( 3 casos) de procedimientos frustros y $2,91 \%$ (3 casos), donde persistió una estenosis residual $\geq 30 \%$.

Observando una tasa de éxito clínico global de la ATP aortoilíaca del $90,29 \%$, realizando 9 procedimientos de recanalización $(8,74 \%)$, con una tasa de éxito técnico del $88,89 \%$, y de éxito clínico del $77,78 \%$ (Tabla 4).

Los mejores resultados relacionados a la ATP del sector aortoíliaco, los presentó el grupo ATP con stent, al comparar a los grupos ATP stent vs ATP simple (Tablas 4 y 5), se observó: éxito técnico del procedimiento $(95,59 \%$ vs $92,30 \%, \mathrm{p}>0,05)$, éxito clínico $(91,18 \%$ vs $84,61 \%, \mathrm{p}>0,05)$, permeabilidad primaria a 5 años $(72,05 \%$ vs $61,53 \%$, p $<0,05)$, permeabilidad primaria asistida a 5 años $(77,94 \%$ vs $69,23 \%, \mathrm{p}>0,05)$, y permeabilidad secundaria a 5 años $(83,82 \%$ vs $76,92 \%, \mathrm{p}>0,05)$.

La morbilidad global relacionada al procedimiento (Tabla 6) fue del 9,70\%, destacando la cardiopatía isquémica aguda $(1,94 \%)$, la neumonía intrahospitalaria $(1,94 \%)$ y el deterioro transitorio de la función renal $(1,94 \%)$.

Las complicaciones relacionadas al procedimiento se decriben en la Tabla 6 , destacando la 
disección focal en el sitio de la ATP $(5,83 \%)$, el hematoma en el sitio de punción $(3,88 \%)$, y la embolía distal $(2,92 \%)$. Todas las disecciónes fueron autolimitadas, ocurriendo durante la predilatación de lesiones de altogrado, siendo tratadas en su totalidad intraprocedimiento implantando un stent, observando adecuada permeabilidad en la angiográfia control final.

En todos los hematomas se realizó manejo médico, presentando en su totalidad resolución espontánea. Las embolías distales fueron manejadas en su totalidad administrando anticoagulación terapéutica, presentando todos los pacientes adecuada evolución, sin complicaciones asociadas.

Dos casos $(1,94 \%)$, presentaron perforación incidental de la arteria iliaca externa, relacionado al intento de cruzar lesiones oclusivas. En ambos casos se procedió al inflado de un balón de angioplastia, proximal a la lesión para controlar el sangrado, y posterior implante en el area de la lesión de un stent cubierto $\left(V^{2} a b a h n^{\circledR}\right.$, W. L. Gore), presentando ambos pacientes adecuada permeabilidad en el control angiográfico final, y óptima evolución sin necesidad de transfundir hemoderivados.

Un caso $(0,97 \%)$, presentó un pseudoaneurisma de $10 \mathrm{~mm}$ en el sitio de punción (arteria femoral común), manejado mediante compresión ecoguiada, logrando su resolución satisfactoria, sin evidenciar secuelas a largo plazo.

En relación a todos los procedimientos de ATP aortoilíaca, el tiempo quirúrgico promedio fue de $95 \mathrm{~min}$, promedio de contraste utilizado $(62,4 \mathrm{cc})$, sangrado promedio (125 cc), estancia hospitalaria promedio de 7,36 días (rango 2-27), con una mortalidad $<30$ días del 1,94\% ( 2 casos), relacionada a cardiopatía isquémica aguda, en pacientes con enfermedad coronaria avanzada.

La permeabilidad primaria global de la ATP aortoilíaca a 5 años (Figura 2), al comparar pacientes con clínica de claudicación $v s$ isquémia crítica, fue del $74,47 \%$ vs $61,7 \%(\mathrm{p}<0,01)$, respectivamente, al comparar lesiones estenosantes vs oclusivas, fue del $70,51 \%$ vs $62,5 \%,(p<0,05)$ respectivamente, y al comparar lesiones localizadas en la ilíaca común vs ilíaca externa del \% 73,33 vs 63,26\% $(\mathrm{p}<0,05)$, respectivamente.

Realizando un seguimiento promedio de 47,13 meses (14-84), obteniendo a 5 años en relación a todos los procedimientos de ATP aortoilíaca, las siguientes tasas globales (Tabla 7): permeabilidad primaria $(68,09 \%)$, permeabilidad primaria asistida $(75,53 \%)$, permeabilidad secundaria $(81,91 \%)$, salvación de la extremidad $(84,04 \%)$, y supervivencia $(90,42 \%)$.
Tabla 4. Tasa de éxito técnico y clínico, relacionada a los distintos procedimientos de angioplastía del sector aortoilíaco

\begin{tabular}{|lccc|}
\hline Procedimiento & n (\%) & $\begin{array}{c}\text { Éxito técnico } \\
\text { n (\%) }\end{array}$ & $\begin{array}{c}\text { Éxito clínico } \\
\text { n (\%) }\end{array}$ \\
ATP + stent & $68(66,02)$ & $65(95,59)$ & $63(92,64)$ \\
ATP simple & $26(25,24)$ & $24(92,30)$ & $23(88,46)$ \\
Recanalización & $9(8,74)$ & $8(88,89)$ & $7(77,78)$ \\
Total & $103(100)$ & $97(94,17)$ & $93(90,29)$ \\
\hline
\end{tabular}

Tabla 5. Permeabilidad primaria, primaria asistida y secundaria, relacionada a los distintos procedimientos de angioplastía del sector aortoilíaco

\begin{tabular}{|lccc|}
\hline Procedimiento & $\mathbf{1}$ año & $\mathbf{3}$ años & $\mathbf{5}$ años \\
Permeabilidad primaria (\%) & & & \\
ATP + stent & 94,11 & 82,53 & 72,05 \\
ATP simple & 88,46 & 73,07 & 61,54 \\
Total & 92,55 & 79,78 & 68,09 \\
Permeabilidad primaria asistida (\%) & & & \\
ATP + stent & 94,11 & 85,29 & 77,94 \\
ATP simple & 88,46 & 80,77 & 69,23 \\
Total & 92,55 & 84,04 & 75,53 \\
Permeabilidad secundaria (\%) & & & \\
ATP + stent & 95,59 & 88,23 & 83,82 \\
ATP simple & 92,30 & 84,61 & 76,92 \\
Total & 94,68 & 87,23 & 81,91 \\
\hline
\end{tabular}

Tabla 6. Complicaciones del tratamiento endovascular de la patología del sector aorto ilíaco $(n=103)$

\begin{tabular}{|lll|}
\hline Complicación & $\mathbf{n}$ & $\mathbf{\%}$ \\
General & & \\
Cardiovascular & 2 & 1,94 \\
Neumonía intrahospitalaria & 2 & 1,94 \\
Deterioro transitorio de la función renal & 2 & 1,94 \\
Ataque isquémico transitorio & 1 & 0,97 \\
Infección del tracto urinario & 1 & 0,97 \\
Trombocitopenia inducida por la heparina & 1 & 0,97 \\
Alergia leve al medio de contraste & 1 & 0,97 \\
Local & & \\
Disección focal en el sitio de la ATP & 6 & 5,83 \\
Hematoma en el sitio de punción & 4 & 3,88 \\
Embolía distal & 3 & 2,92 \\
Espasmo arterial & 2 & 1,94 \\
Perforación arterial & 2 & 1,94 \\
Pseudoaneurisma en el sitio de punción & 1 & 0,97 \\
\hline
\end{tabular}




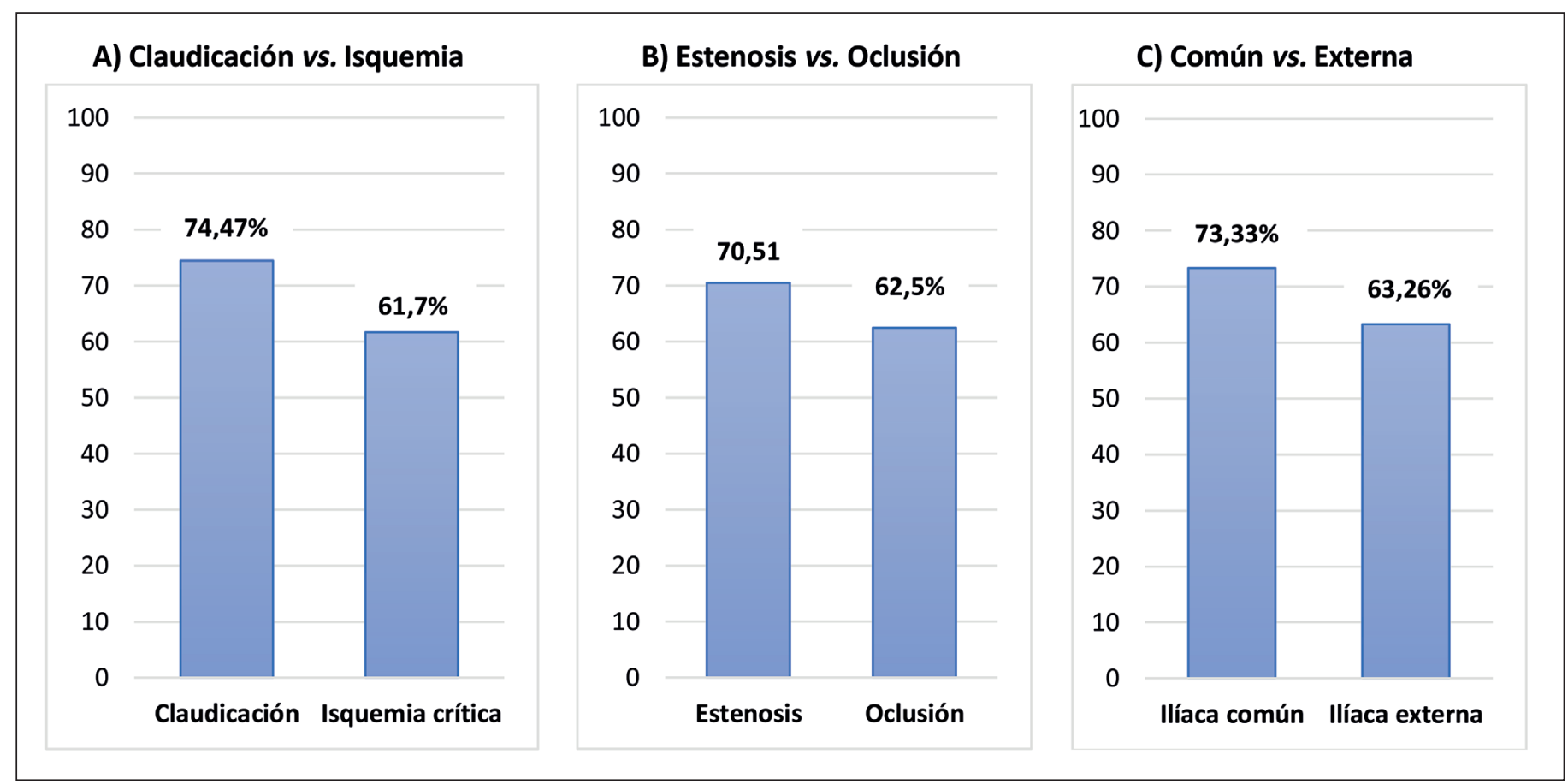

Figura 2. Análisis comparativo de factores clínicos y anatómicos, que influyen en la tasa de permeabilidad primaria global a 5 años, de la ATP aortoilíaca. A) Claudicación vs. Isquemia crítica, B) Estenosis vs. Oclusión, C) Arteria ilíaca común vs. Arteria ilíaca externa.

Tabla 7. Tasa global de permeabilidad primaria, permeabilidad primaria asistida, permeabilidad secundaria, salvación de la extremidad y supervivencia, relacionada a la angioplastia del sector aortoilíaco

\begin{tabular}{|lccc|}
\hline Tasas & 1 año (\%) & 3 años (\%) & $\mathbf{5}$ años (\%) \\
Permeabilidad primaria & 92,55 & 79,78 & 68,09 \\
Permeabilidad primaria asistida & 92,55 & 84,04 & 75,53 \\
Permeabilidad secundaria & 94,68 & 87,23 & 81,91 \\
Salvación de la extremidad & 95,74 & 90,42 & 84,04 \\
Supervivencia & 97,87 & 93,61 & 90,42 \\
\hline
\end{tabular}

\section{Discusión}

El éxito técnico de la ATP ilíaca en lesiones focales, es superior al $90 \%$, y del $80-85 \%$ en lesiones difusas $^{17,18}$, con permeabilidad primaria superior al $70 \%$ a 5 años, en casi todas las series ${ }^{17-19}$, observando un éxito técnico del $92 \%$ (50-96), con permeabilidad global a dos años del $81 \%$ (65-93), y a cinco años del $72 \%(50-87)^{18-20}$, la gran disparidad entre las series, puede explicarse en las diferencias de la población estudiada, y métodos de análisis utilizados.
Nuestros resultados son similares a las series publicadas $^{17-20}$, evidenciando una tasa de éxito técnico global del $94,17 \%$, y permeabilidad primaria global a 5 años del 68,09\%. Sin embargo la mayoría de las series evaluan el resultado de la ATP, al tratar lesiones TASC II tipo A y B, considerando en nuestra serie lesiones con resultados menos favorables como las TASC II C y D, obteniendo igualmente buenos resultados (permeabilidad primaria a 5 años del $50 \%$ y $40 \%$, respectivamente), permitiendo así simplificar los procedimientos de revascularización más distal mejorando el inflow, o acompañando a otros procedimientos de revascularización infrainguinal.

De igual forma con la ATP, mantenemos la posibilidad de recanalización de la zona angioplastiada en caso de reestenosis, obteniendo en nuestra serie una elevada permeabilidad primaria secundaria global a 5 años del $81,91 \%$. No impidiendo la posterior revascularización mediante cirugía convencional en caso de ser necesaria ${ }^{19,21}$.

Los mejores resultados de la ATP aortoilíaca en nuestro estudio, se observan en pacientes con claudicación, estenosis, lesiones TASC II tipo A y $\mathrm{B}$, lesiones localizadas en la arteria ilíaca común, y en la ATP con stent. Por el contrario los resultados menos favorables, se observaron en pacientes con 
isquemia crítica, lesiones oclusivas, lesiones TASC II C y D, lesiones localizadas en la arteria iliaca externa, y en la ATP simple.

Hallazgos que concuerdan con la literatura $^{18,19,21,22}$, donde se reporta que existen múltiples factores que afectan negativamente la tasa de éxito, y permeabilidad a largo plazo de la ATP, como el sexo femenino, diabetes mellitus, enfermedad renal crónica, isquemia crítica, lesión oclusiva, lesión localizada en la arteria ilíaca externa, longitud de la lesión $>10 \mathrm{~cm}$, y mal runoff.

Así en la sitiación más desfavorable (isquemia crítica, oclusión extensa de la ilíaca externa, con mal run-off ), la permeabilidad primaria a cinco años es del $6 \%$, frente al $63 \%$ en el caso más favorable (claudicador, con estenosis segmentaria única de la ilíaca común, y buen run-off) ${ }^{22-24}$.

Los mejores resultados a largo plazo de nuestra serie, los obtuvo la ATP con stent, observando al comparar la ATP simple vs ATP con stent, una permeabilidad primaria a 5 años del $72,05 \%$ vs $61,53 \%,(p<0,05)$ respectivamente. Sin embargo el resto de resultados observados al comparar ambos procedimientos, no fueron estadisticamente significativos, pudiendo explicarse en un sesgo de selección, debido a que la mayoría de las lesiones tratadas con stent, son más complejas (lesiones largas, gravemente calcificadas, oclusiones, disecciónes, entre otros), y con pronóstico más desfavorable a largo plazo.

El uso de stent primario en lesiones poco favorables (gravemente calcificadas, excéntricas, ulceradas, extensa longitud), ${ }^{25}$ obtiene buenos resultados, con éxito técnico del $95 \%$, permeabilidad primaria del $87 \%$ al año, y mayor del $54 \%$ a cinco años ${ }^{25,26}$.

Al comparar la ATP con stents vs ATP simple, se observa una permeabilidad primaria estimada a cuatro años del $64 \%$ para ATP simple, y del 77\% para ATP con stent ${ }^{26,27}$ con una disminución del riesgo relativo de fallo del procedimiento a largo plazo del $43 \%$, mediante la utilización de stent ${ }^{28}$.

Los stent mejoran la permeabilidad del vaso, añadiendo soporte a la tensión radial de la pared, evitando el rebote elástico, favoreciendo la correcta remodelación arterial, logrando comprimir disecciones de la íntima, disminuyendo el proceso de reestenosis debida a hiperplasia intimal, o por progresión de la enfermedad ${ }^{26-28}$.

Los stent balón expandibles presentan mayor fuerza radial y precisión durante el despliegue, recomendando su uso a nivel aórtico, bifurcación aórtica, arteria ilíaca común y en técnicas tipo kissing balloon ${ }^{29,30}$. Los stent autoexpandibles al presentar menor perfil, ser más flexibles y nave- gables, se adaptan mejor a anatomías tortuosas y diferentes diámetros arteriales, recomendando su uso en lesiones extensas, y en casos de stenting contralateral $^{29-31}$.

Las principales limitaciones para la realización de las técnicas endovasculares se deben a la tortuosidad, calcificación, morfología y extensión de la lesión aortoilíaca a tratar ${ }^{32}$. La incorrecta indicación, o uso inadecuado de materiales, determinan el fracaso, y probable aparición de lesiones iatrogénicas ${ }^{33,34}$. Es fundamental conocer la anatomía del territorio a tratar, materiales disponibles en la actualidad, y contar con formación especializada ${ }^{32-34}$.

Una crítica del presente trabajo, es la ausencia de evalución de la calidad de vida de los pacientes, sometidos al tratamiento endovascular. Son necesarios ensayos prospectivos, aleatorizados, multicéntricos, que evaluen la calidad de vida de los pacientes, comparando la ATP simple y con stent a largo plazo, y estudios que comparen los resultados entre la cirugía convencional y la endovascular.

\section{Conclusiones}

En pacientes adecuadamente seleccionados, el tratamiento endovascular del sector aortoilíaco presenta excelentes resultados, permitiendo aumentar la indicación de tratamiento en pacientes considerados de alto riesgo.

Los mejores resultados de la ATP aortoilíaca, se obtienen en pacientes con claudicación, lesiones estenosantes, lesiones TASC II tipo A y B, lesiones localizadas en la arteria ilíaca común, y en la ATP con stent (especialmente en lesiones severamente calcificadas).

Los procedimientos híbridos representan una excelente estratégia, en pacientes considerados de alto riesgo, con excelentes resultados y reducción significativa de la estancia hospitalaria, con alto grado de tolerancia por parte del paciente.

\section{Responsabilidades éticas}

Protección de personas y animales. Los autores declaran que para esta investigación no se han realizado experimentos en seres humanos ni en animales.

Confidencialidad de los datos. Los autores declaran que en este artículo no aparecen datos de pacientes.

Conflictos de interés: no hay. 


\section{Bibliografía}

1. Eraso LH, Fukaya E, Mohler ER, Xie D, Sha D, Berger JS. Peripheral arterial disease, prevalence and cumulative risk factor profile analysis. Eur J Prev Cardiol. 2014;21:704-11.

2. Dua A, Lee CJ. Epidemiology of Peripheral Arterial Disease and Critical Limb Ischemia. Tech Vasc Interv Radiol. 2016;19:91-5.

3. Nehler MR, Duval S, Diao L, Annex BH, Hiatt WR, Roger K, et al. Epidemiology of peripheral arterial disease and critical limb ischemia in an insured national population. J Vasc Surg. 2014;60:686-95. e2.

4. Norgren L, Hiatt WR, Dormandy JA, Nehler MR, Harris KA, Fowkes FGR, et al. Inter-Society Consensus for the Management of Peripheral Arterial Disease (TASC II). J Vasc Surg. 2007;45:S5-S67.

5. Gerhard-Herman MD, Gornik HL, Barrett C, Barshes NR, Corriere MA, Drachman DE, et al. 2016 AHA/ACC Guideline on the Management of Patients With Lower Extremity Peripheral Artery Disease: Executive Summary: A Report of the American College of Cardiology/ American Heart Association Task Force on Clinical Practice Guidelines. J Am Coll Cardiol. 2017;69:1465-508.

6. Bracale UM, Giribono AM, Spinelli D, Del Guercio L, Pipitò N, Ferrara D, et al. Long-term Results of Endovascular Treatment of TASC C and D Aortoiliac Occlusive Disease with Expanded Polytetrafluoroethylene Stent Graft. Ann Vasc Surg. 2019;56:254-60.

7. Ray JJ, Eidelson SA, Karcutskie CA, Meizoso JP, DeAmorim H, Goldstein LJ, et al. Hybrid Revascularization Combining Iliofemoral Endarterectomy and Iliac Stent Grafting for TransAtlantic Inter-Society Consensus $\mathrm{C}$ and D Aortoiliac Occlusive Disease. Ann Vasc Surg. 2018;50:73-9.

8. Dosluoglu HH. Commentary: endovascular therapy should be the first line of treatment in patients with severe (TASC II C or D) aortoiliac occlusive disease. J Endovasc Ther. 2013;20:74-9.

9. Suzuki K, Mizutani Y, Soga Y, Iida O, Kawasaki D, Yamauchi Y, et al. Efficacy and Safety of Endovascular Therapy for
Aortoiliac TASC D Lesions. Angiology 2017;68:67-73.

10. Dosluoglu HH, Lall P, Cherr GS, Harris LM, Dryjski ML. Role of simple and complex hybrid revascularization procedures for symptomatic lower extremity occlusive disease. J Vasc Surg. 2010;51:1425-35.e1.

11. Huynh TT, Bechara CF. Hybrid interventions in limb salvage. Methodist Debakey Cardiovasc J. 2013;9:90-4.

12. Uhl C, Betz T, Weiss B, Töpel I, Steinbauer M. Results of hybrid procedures for treatment of aortoiliac Trans-Atlantic Inter-Society Consensus II D lesions with self-expanding covered heparin-bonded stent grafts. J Cardiovasc Surg. (Torino) 2020;61:93-7.

13. Burke CR, Henke PK, Hernandez R, Rectenwald JE, Krishnamurthy V, Englesbe MJ, et al. A contemporary comparison of aortofemoral bypass and aortoiliac stenting in the treatment of aortoiliac occlusive disease. Ann Vasc Surg. 2010;24:4-13.

14. Piffaretti G, Fargion AT, Dorigo W, Pulli R, Gattuso A, Bush RL, et al. ILIACS Registry Group. Outcomes From the Multicenter Italian Registry on Primary Endovascular Treatment of Aortoiliac Occlusive Disease. J Endovasc Ther. 2019;26:623-32.

15. Schmalstieg J, Zeller T, Tübler T, Sixt S, Schwencke C, Sandstede J, et al. Long term data of endovascularly treated patients with severe and complex aortoiliac occlusive disease. J Cardiovasc Surg. (Torino) 2012;53:291-300.

16. Kashyap VS, Pavkov ML, Bena JF, Sarac TP, O'Hara PJ, Lyden SP, et al. The management of severe aortoiliac occlusive disease: endovascular therapy rivals open reconstruction. J Vasc Surg. 2008 Dec;48(6):1451-7, 1457.e1-3.

17. Jongkind V, Akkersdijk GJ, Yeung KK, Wisselink W. A systematic review of endovascular treatment of extensive aortoilíac occlusive disease. J Vasc Surg. 2010;52:1376-83.

18. Yuan L, Bao J, Zhao Z, Feng X, Lu Q, Jing, Z. Endovascular therapy for long-segment atherosclerotic aortoiliac occlusion. Journal of Vascular Surgery 2014; 59:663-8

19. Ozkan U, Oguzkurt L, Tercan F. Technique, complication, and long-term outcome for endovascular treatment of iliac artery occlusion. Cardiovascular and Interventional Radiology 2010;33:18-24.

20. Aggarwal V, Waldo SW, Armstrong EJ. Endovascular revascularization for aortoiliac atherosclerotic disease. Vasc Health Risk Manag. 2016;12:117-27.

21. Indes JE, Mandawat A, Tuggle CT, Muhs B, Sosa JA. Endovascular procedures for aorto-iliac occlusive disease are associated with superior short-term clinical and economic outcomes compared with open surgery in the inpatient population. J Vasc Surg. 2010;52:1173-9, 1179.e1.

22. Pulli R, Dorigo W, Fargion A, Innocenti A, Pratesi G, Marek J, et al. Early and long-term comparison of endovascular treatment of iliac artery occlusions and stenosis. J Vasc Surg. 2011;53:92-8.

23. Ichihashi $\mathrm{S}$, Higashiura $\mathrm{W}$, Itoh $\mathrm{H}$, Sakaguchi S, Nishimine K, Kichikawa $\mathrm{K}$. Long-term outcomes for systematic primary stent placement in complex iliac artery occlusive disease classified according to Trans-Atlantic Inter-Society Consensus (TASC)-II. J Vasc Surg. 2011;53:992-9.

24. Davies MG, Bismuth J, Saad WE, Naoum JJ, Peden EK, Lumsden AB. Outcomes of reintervention for recurrent disease after percutaneous iliac angioplasty and stenting. J Endovasc Ther. 2011;18:16980.

25. Tetteroo E, van der Graaf Y, Bosch JL, van Engelen AD, Hunink MG, Eikelboom BC, et al. Randomised comparison of primary stent placement versus primary angioplasty followed by selective stent placement in patients with iliac-artery occlusive disease. Dutch Iliac Stent Trial Study Group. Lancet. 1998;351(9110):1153-9.

26. Klein WM, van der Graaf Y, Seegers J, Spithoven JH, Buskens E, van Baal JG, et al. Dutch iliac stent trial: long-term results in patients randomized for primary or selective stent placement. Radiology. 2006;238:734-44.

27. Bekken J, Jongsma H, Ayez N, Hoogewerf CJ, Van Weel V, Fioole B. Angioplasty versus stenting for iliac artery lesions. Cochrane Database Syst Rev. 2015;5:CD007561.

28. Goode SD, Cleveland TJ, Gaines PA; STAG trial collaborators. Randomized clinical trial of stents versus angioplasty 
for the treatment of iliac artery occlusions (STAG trial). Br J Surg. 2013;10:1148-53.

29. Bekken JA, Jongsma H, de Vries JP, Fioole B. Self-expanding stents and aortoiliac occlusive disease: a review of the literature. Med Devices (Auckl). 2014;7:99-105.

30. Krankenberg H, Zeller T, Ingwersen M, Schmalstieg J, Gissler HM, Nikol S, et al. Self-Expanding Versus BalloonExpandable Stents for Iliac Artery Occlusive Disease: The Randomized ICE Trial. JACC Cardiovasc Interv.
2017;10:1694-704.

31. Mwipatayi BP, Sharma S, Daneshmand

A, Thomas SD, Vijayan V, Altaf $\mathrm{N}$ et al. Durability of the balloon-expandable covered versus bare-metal stents in the Covered versus Balloon Expandable Stent Trial (COBEST) for the treatment of aortoiliac occlusive disease. J Vasc Surg. 2016;64:83-94.e1.

32. Müller AM, Langwieser N, Bradaric C, Haller B, Fusaro M, Ott I, et al. Endovascular Treatment for StenoOcclusive Iliac Artery Disease: Safety and Long-Term Outcome. Angiology 2018;69:308-15.

33. Mayor J, Branco BC, Chung J, MonteroBaker MF, Kougias P, Mills JL, et al. Outcome Comparison between Open and Endovascular Management of TASC II D Aortoiliac Occlusive Disease. Ann Vasc Surg. 2019;61:65-71.e3.

34. Suzuki K, Mizutani Y, Soga Y, Iida O, Kawasaki D, Yamauchi Y, et al. Efficacy and Safety of Endovascular Therapy for Aortoiliac TASC D Lesions. Angiology 2017;68:67-73 